\title{
A dynamic Monte Carlo study of anomalous current voltage behaviour in organic solar cells
}

\author{
K. Feron, ${ }^{1,2, a)}$ X. Zhou, ${ }^{1}$ W. J. Belcher, ${ }^{1}$ C. J. Fell, ${ }^{1,2}$ and P. C. Dastoor ${ }^{1}$ \\ ${ }^{1}$ Centre for Organic Electronics, University of Newcastle, Callaghan, NSW 2308, Australia \\ ${ }^{2}$ CSIRO Energy Flagship, Newcastle, NSW 2300, Australia
}

(Received 3 October 2014; accepted 23 November 2014; published online 5 December 2014)

\begin{abstract}
We present a dynamic Monte Carlo (DMC) study of s-shaped current-voltage (I-V) behaviour in organic solar cells. This anomalous behaviour causes a substantial decrease in fill factor and thus power conversion efficiency. We show that this s-shaped behaviour is induced by charge traps that are located at the electrode interface rather than in the bulk of the active layer, and that the anomaly becomes more pronounced with increasing trap depth or density. Furthermore, the s-shape anomaly is correlated with interface recombination, but not bulk recombination, thus highlighting the importance of controlling the electrode interface. While thermal annealing is known to remove the s-shape anomaly, the reason has been not clear, since these treatments induce multiple simultaneous changes to the organic solar cell structure. The DMC modelling indicates that it is the removal of aluminium clusters at the electrode, which act as charge traps, that removes the anomalous I-V behaviour. Finally, this work shows that the s-shape becomes less pronounced with increasing electron-hole recombination rate; suggesting that efficient organic photovoltaic material systems are more susceptible to these electrode interface effects. ( 2014 AIP Publishing LLC.
\end{abstract}

[http://dx.doi.org/10.1063/1.4903530]

\section{INTRODUCTION}

Many organic solar cell models, such as drift-diffusion type or electrical equivalent circuit models, provide a good fit against experimental data when organic photovoltaic (OPV) devices with well-behaved current-voltage (I-V) curves are modelled. ${ }^{1,2}$ However, these computational techniques do not readily reproduce the anomalous I-V characteristics that are observed experimentally. As such, new approaches to modelling these organic solar cells are required to understand and ultimately prevent this undesirable non-ideal behaviour.

A well known anomaly in I-V measurements of organic solar cells is the appearance of a "kink" or s-shape., ${ }^{3,4}$ Two examples of such an anomaly are shown in Figure 1, together with a normal I-V curve. Generally, short-circuit current density $\left(J_{S C}\right)$ and open-circuit voltage $\left(V_{O C}\right)$ are marginally affected by the s-shape effect, in contrast to fill factor $(F F)$ which is severely reduced. While devices that exhibit s-shaped $\mathrm{I}-\mathrm{V}$ curves can be deliberately fabricated, ${ }^{5}$ the opposite is not always true. Even though the same fabrication recipe is used, a device that exhibits s-shaped I-V behaviour is observed from time to time. ${ }^{6}$

The occurrence of s-shape behaviour has been linked to vertical phase segregation in OPV devices with atomic force microscopy (AFM) and I-V measurements showing that the metal-organic interface is especially important, given that devices with a Poly(3-hexylthiophene) (P3HT) rich cathode-active layer interface are much more prone to s-shape behaviour. ${ }^{6}$ In fact, Tremolet de Villers et al. ${ }^{6}$ showed that s-shape behaviour can be prevented by the deposition of a thin PCBM layer on

\footnotetext{
${ }^{\text {a) }}$ Author to whom correspondence should be addressed. Electronic mail: Krishna.Feron@csiro.au
}

top of the bulk heterojunction (BHJ) active layer (cathode side). S-shaped I-V curves commonly arise in pristine devices and often disappear after thermal annealing treatments. ${ }^{7}$ Since annealing treatments are known to modify the vertical distribution of PCBM, ${ }^{8-11}$ it is possible that the disappearance of the kink in I-V curves after annealing is simply the result of increased PCBM concentration at the cathode interface. De Castro et al. ${ }^{12}$ have shown that the insertion of a thin bathocuproine $(\mathrm{BCP})$ layer in between the active layer and cathode also prevents s-shape behaviour. However, if high aluminium deposition rates are used, a kink in the I-V curves is again observed despite the BCP layer. Certain interface layers, such as BCP, are believed to prevent physical destruction of the organic layer when the metal cathode is vacuum deposited. ${ }^{3,13,14}$ De Castro et al. propose that isolated aluminium nanoclusters are formed as a result of metal atom diffusion during the evaporative deposition process. These clusters are believed to act as charge carrier trapping sites. The authors also propose that these aluminium clusters subsequently coalesce at elevated temperature (thermal annealing) and merge with the bulk cathode, thus removing the trapping sites. Wagner $\mathrm{et} \mathrm{al.}^{3}$ showed that samarium, when used in place of aluminium, does not penetrate into the underlying organic layer and forms a continuous film after deposition of a few monolayers. When using samarium, BCP is not needed to prevent s-shaped I-V curves. This observation provides further evidence that the s-shape behaviour is related to aluminium diffusion into the organic layer.

However, it is not just the electrode-organic interface that governs the presence of s-shaped I-V curves. Tress et al. ${ }^{15}$ showed that materials systems with a mobility mismatch larger than a factor of 100 exhibit s-shape I-V curves when a bilayer architecture is used due to asymmetric charge distribution, which significantly disrupts the internal electric field. In a BHJ structure, no s-shaped curves were observed, 


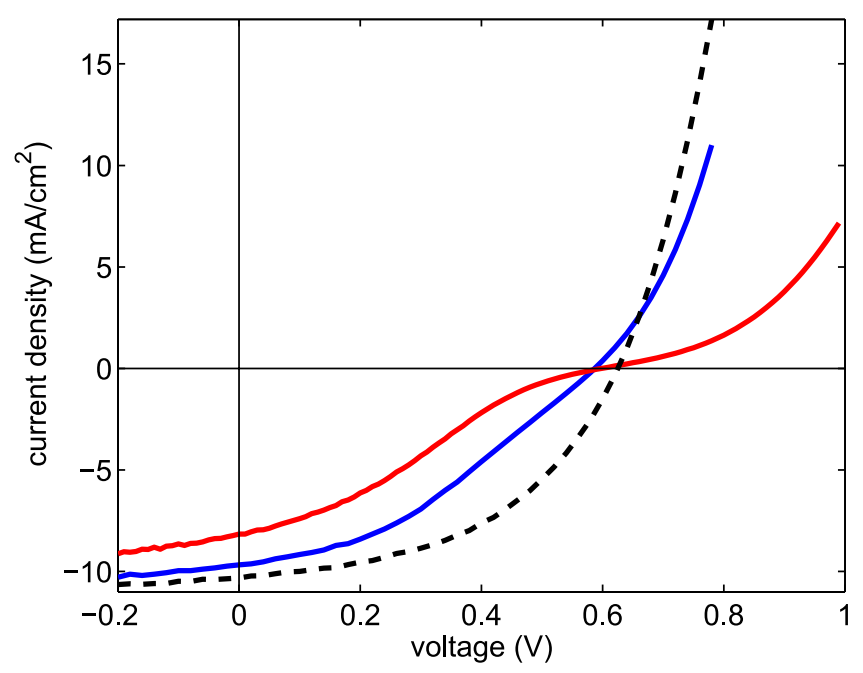

FIG. 1. Three measured I-V curves corresponding to Al/P3HT:PCBM/ PEDOT:PSS/ITO devices. The dashed line is a normal I-V curve, while the red and blue solid lines are s-shaped I-V curves. The red curve shows a much more severe kink than the blue curve.

because both donor and acceptor material are dispersed throughout the active layer, which results in spatially uniform charge distribution (and thus electric field) in the active layer.

The most frequently used modelling approaches for organic solar cells include electrical equivalent circuit models and drift-diffusion models. In both cases, these models have to be significantly modified to fit s-shaped curves. For example, the standard one-diode electrical equivalent circuit model cannot produce s-shaped curves ${ }^{16}$ and an extra diode in reverse bias with an associated parallel resistance has to be introduced. $^{12}$ Ecker et $a .^{4}$ proposed an entirely different equivalent circuit model based on impedance spectroscopy measurements. While good fits could be obtained with these equivalent circuit models, they do not allow for a specific investigation of charge carrier trap distributions and often lack a clear physical connection between fitting parameters and real device conditions. Drift-diffusion type models have also been adapted in order to describe anomalous I-V behaviour. Kumar et al. ${ }^{17}$ proposed a model where s-shaped behaviour is induced by interface dipoles with a corresponding electric field that points in the opposite direction to the built-in field. Wagenpfahl et al. ${ }^{5}$ took a more general approach to charge extraction and introduced a semiconductor-electrode surface recombination term, which models restrictions on the charge extraction process. Interestingly, both of the modifications implemented by Kumar et al. and Wagenpfahl et al. are related to the active layer-electrode interface and were able to more successfully model s-shaped I-V curves.

From the preceding discussion, it is clear that nature of the cathode-organic interface can influence the creation of s-shaped I-V curves via two possible mechanisms: (1) vertical phase segregation of PCBM and (2) induced trapping sites near the cathode. Furthermore, both effects are not mutually exclusive and can occur simultaneously upon thermal annealing, which makes it difficult to isolate the effect of each mechanism experimentally. Charge traps can be caused by impurities or defects, either at the electrode interface or in the bulk of the active layer. Such defects are hard to control and may be the result of poor fabrication procedures, interface materials (e.g., calcium) penetrating into the organic layers and/or giving rise to gap states, ${ }^{18}$ electrode penetration into the active layer, ${ }^{12,19}$ the use of insufficiently pure material, ${ }^{3}$ or degradation of any of the components in an OPV device. ${ }^{20}$ Additionally, small isolated islands of electron donor and/or acceptor material can effectively act as charge trapping regions. ${ }^{20}$ While the existence of charge traps is well known, their simulated impact on the shape of $\mathrm{I}-\mathrm{V}$ curves is only briefly discussed in the literature. Mandoc et $a .^{21}$ investigated electron traps in poly[2-methoxy-5(3',7'-dimethyloctyloxy)-1,4-phenylene vinylene] (MDMOPPV) devices using a 1D drift-diffusion based modelling approach and although they did not report evidence for sshaped curves; they did show that $V_{O C}, J_{S C}, F F$ and thus power conversion efficiency $(P C E)$ are negatively influenced by electron traps due to increased charge recombination. On the other hand, Giebink et al. ${ }^{22}$ used drift-diffusion based modelling to show that electron traps can cause s-shaped I-V curves.

The observed relation between traps and s-shaped I-V behaviour warrants deeper investigation and the development of a computational modelling approach that is capable of probing directly the physical processes taking place. The dynamic Monte Carlo (DMC) approach allows for comprehensive, accurate mesoscopic models with a full consideration of the three-dimensional structure of the active layer. ${ }^{11,23,24}$ However, despite experimental validation of the increasingly popular DMC approach, ${ }^{11,25}$ S-shaped I-V behaviour has not been investigated using a DMC model. Here, we use a DMC model to investigate the isolated effect of vertical phase segregation on the I-V curve shape and the effect of charge carrier trapping sites on the occurrence of s-shaped I-V curves. In particular, we investigate the impact of the location of charge traps in relation to s-shaped I-V curves, the link between charge recombination and s-shape severity and we also show how light intensity influences the apparent s-shape.

\section{EXPERIMENTAL AND SIMULATION METHODOLOGY}

\section{A. Device fabrication}

Devices were fabricated based on the structure indium tin oxide (ITO)/poly(3,4-ethylenedioxythiophene):poly(styrenesulfonate) (PEDOT:PSS)/P3HT:[6,6]-phenyl-C61-butyric acid (PCBM)/Aluminium (Al). Patterned ITO on glass substrates was cleaned using a series of solvent sonication followed by a 15 min plasma treatment in a mixture of air and oxygen. A $40 \mathrm{~nm}$ PEDOT:PSS layer was deposited via spin coating, followed by a 10 min thermal treatment in air. A 1:1 blend of P3HT:PCBM was dissolved in chlorobenzene and spincast on top of the PEDOT:PSS layer under a nitrogen atmosphere. Finally, an aluminium cathode is deposited via evaporation to complete the OPV device. Devices were annealed at $140^{\circ} \mathrm{C}$ for $4 \mathrm{~min}$.

\section{B. Device characterisation}

I-V characteristics of OPV devices were measured under simulated sunlight (Newport Oriel 91160 with Class A match 
to the AM1.5 reference spectrum). The light intensity was varied using neutral density filters and was measured using a calibrated silicon reference solar cell (Fraunhofer ISE).

\section{Dynamic Monte Carlo model}

The DMC model has been described previously. ${ }^{11,26}$ In short, a three dimensional Cartesian lattice is created with a voxel size of $1 \times 1 \times 1 \mathrm{~nm}$. Each voxel represents electron donor or acceptor material. The arrangement of donor and acceptor material is determined by a cellular automata method $^{26,27}$ and is chosen to mimic a bulk heterojunction morphology. The reader is referred to supplementary material for more information on the simulated BHJ morphologies. ${ }^{28}$ A first reaction method is employed to calculate reaction rates, $k$. Rates are converted to waiting times, $t$, through

$$
t=\frac{-\log (X)}{k},
$$

where $\mathrm{X}$ is a random number between 0 and 1 . Exciton generation (i.e., light absorption) rates as a function of depth in the active layer are assigned according to the optical field of a P3HT:PCBM device as determined through a transfer matrix method. ${ }^{29}$ Exciton hopping rates are calculated following Förster resonance energy transfer (FRET) theory, ${ }^{30}$ using

$$
k_{F R E T}=\frac{1}{t_{0}}\left(\frac{R_{0}}{R_{i j}}\right)^{6} f\left(E_{i}, E_{j}\right),
$$

where $t_{0}$ is the exciton lifetime, $R_{0}$ the Förster radius, $R_{i j}$ the distance between hopping sites, $E_{\mathrm{i}}$ the energy of the ith site, $\mathrm{E}_{\mathrm{j}}$ the energy of the jth site, and $f\left(E_{i}, E_{j}\right)$ the Boltzmann factor, which is given by

$$
f\left(E_{i}, E_{j}\right)= \begin{cases}e^{-\frac{E_{j}-E_{i}}{k_{B} T}}, & E_{j}>E_{i} \\ 1, & E_{j}<E_{i} .\end{cases}
$$

Here, $\mathrm{k}_{\mathrm{B}}$ is Boltzmann's constant and $\mathrm{T}$ is temperature. Excitons can recombine according to their lifetime. If an exciton hops to a donor-acceptor interface it dissociates, thus giving rise to a charge transfer state. In this state, the electron resides on the acceptor and hole on the donor. When electron and hole are adjacent to each other, they can recombine with a rate of $k_{\text {rec }}$. Charge hopping rates are calculated according to Marcus theory ${ }^{31}$ using

$$
k_{\text {Marcus }}=k_{0} \exp \left(-\frac{\left(E_{j}-E_{i}+\lambda\right)^{2}}{4 \lambda k_{B} T}\right),
$$

where $k_{0}$ is the charge hopping rate constant and $\lambda$ the reorganisation energy.

The site energy for charge carriers includes the energy associated with the electric field (both as a result of an externally applied field and the built-in field), $E_{f}$, Coulomb interaction with other charge carriers and image charges (surface charge at the electrodes induced by approaching charge carriers), $E_{c}$, and Gaussian energetic disorder with standard deviation $(\sigma), E_{\sigma}$.

$$
\Delta E_{i j}=\Delta E_{f}+\Delta E_{c}+\Delta E_{\sigma} .
$$

The potential energy difference due to the electric field is calculated using the following equation:

$$
\Delta E_{f}=q \frac{z_{i j}}{Z}\left(V_{e x t}+V_{b i}\right) .
$$

Here, $q$ is the charge of an electron, $z_{i j}$ the vertical distance between sites $i$ and $j, Z$ the thickness of the active layer, $V_{\text {ext }}$ the externally applied voltage, and $V_{b i}$ the built-in voltage due to the work function difference of the electrode. The latter two voltages are dropped over the thickness of the active layer. The Coulomb interaction between (image) charge carriers is calculated using

$$
\Delta E_{c}=\frac{q^{2}}{4 \pi \varepsilon_{0} \varepsilon_{r}} \sum_{r_{i j} \leq r_{c}} \frac{1}{r_{i j}},
$$

where $\varepsilon_{0}$ is the permittivity of free space, $\varepsilon_{r}$ the dielectric constant, $r_{i j}$ the distance between the charge carriers, and $r_{c}$ the Coulomb capture radius. ${ }^{23}$ This cut-off approximation for the Coulomb interaction has been shown to be valid under normal operating conditions. ${ }^{32}$ We assessed the validity of the cut-off approximation for the simulation of $\mathrm{s}$-shaped I-V curves. For typical charge carrier densities $\left(10^{21}-10^{24} \mathrm{~m}^{-3}\right)$ as would be found in P3HT:PCBM devices exposed to AM1.5 light at $1000 \mathrm{~W} / \mathrm{m}^{2}$, no statistical difference in the I-V curves was found for a cut-off radius $\geq 15 \mathrm{~nm}$. Since $r_{c}$ was $19 \mathrm{~nm}$, the use of the cut-off approximation is justified. The reader is referred to the supplementary material for more information on the cut-off approximation. $^{28}$ The energy landscape for excitons is solely determined by energetic disorder, because excitons are a neutral species and are thus assumed not to be affected by electric potential effects. Charge injection is modelled using Miller-Abrahams expression ${ }^{31}$

$$
k_{M A}=k_{i n j} \begin{cases}e^{-\frac{E_{j}-E_{i}}{k_{B} T}}, & E_{j}>E_{i} \\ 1, & E_{j}<E_{i} .\end{cases}
$$

Here, $k_{i n j}$ is a constant determined by the electrode-organic layer injection rate prefactor and the hopping rate prefactor. The extraction barrier for holes at the anode and electrons at the cathode is chosen to be the same and is given by HOMO minus the work function and LUMO minus the work function, respectively. All parameters of the DMC model are given in Table I. We conducted a general investigation of sshaped I-V curves and do not model any specific material system. However, since the balance between injected and photo-generated charge carriers plays a role in the severity in the s-shape effect (as discussed in Sec. II C) it is important to use realistic charge injection parameters (i.e., energy barrier at electrode and $k_{i n j}$ ). The charge injection parameters were chosen to match the simulated dark I-V curve to that of a real P3HT:PCBM device. 
TABLE I. Parameters used in Monte Carlo simulations unless stated otherwise in text.

\begin{tabular}{|c|c|c|}
\hline Property & Value & Reference \\
\hline Temperature, $T$ & $300 \mathrm{~K}$ & \\
\hline Relative dielectric constant, $\varepsilon_{r}$ & 3.1 & 33 \\
\hline Standard deviation Gaussian density of states P3HT and PCBM, $\sigma$ & $0.05 \mathrm{eV}$ & 11 \\
\hline Forster radius, $R_{0}$, acceptor moiety & $3.65 \mathrm{~nm}$ (equivalent to $1 \mathrm{D}$ diffusion length of $40 \mathrm{~nm}$ ) & $34,\left(\mathrm{C}_{60}\right)$ \\
\hline Forster radius, $R_{0}$, donor moiety & $2.15 \mathrm{~nm}$ (equivalent to 1D diffusion length of $8.5 \mathrm{~nm}$ ) & $35,(\mathrm{P} 3 \mathrm{HT})$ \\
\hline Exciton lifetime, $t_{0}$, acceptor moiety & $11 \mu \mathrm{s}$ & 36, (PCBM lifetime) \\
\hline Exciton lifetime, $t_{0}$, donor moiety & $400 \mathrm{ps}$ & 35, P3HT lifetime \\
\hline Charge injection rate at both electrode interface, $k_{i n j}$ & $1.1111 \times 10^{8} \mathrm{~s}^{-1}$ & Fit against dark IV curve \\
\hline Charge hopping rate constant, $k_{0}$, acceptor moiety & $3 \times 10^{12} \mathrm{~s}^{-1}$ & 37 and 38 \\
\hline Charge hopping rate constant, $k_{0}$, donor moiety & $3 \times 10^{12} \mathrm{~s}^{-1}$ & 37 and 38 \\
\hline Charge carrier recombination rate, $k_{r e c}$ & $1 \times 10^{4} \mathrm{~s}^{-1}$ & 11 \\
\hline Reorganization energy, $\lambda$ & $0.5 \mathrm{eV}$ & 39 \\
\hline Lattice constant & $1 \mathrm{~nm}$ & 38 \\
\hline Anode work function & $-4.8 \mathrm{eV}$ & 40, ITO \\
\hline Cathode work function & -4.3 & $40, \mathrm{Al}$ \\
\hline HOMO donor moiety & -5.2 & Similar to P3HT \\
\hline LUMO acceptor moiety & -4.48 & Similar to PCBM \\
\hline
\end{tabular}

As shown in Fig. 2(a), a good fit was obtained as the measured and simulated values agree to within the standard deviation of the simulated values except for the measurement at the highest voltage $(0.9 \mathrm{~V})$. Good agreement was also found under light conditions with again the data point at the highest voltage deviating the most (Fig. 2(b)). The discrepancy of the last point may be the result of not taking into account the series resistance of electrodes leading to a significant overestimation of the measured value at high current densities where the relative impact of resistance is high.

Mobility studies of P3HT:PCBM systems have found evidence for the existence of deep energy traps leading to trapped electrons and holes. ${ }^{41}$ The energy distribution of charge carrier traps have been reported to be exponential ${ }^{42}$ or Gaussian ${ }^{43}$ or neither. ${ }^{20}$ As discussed, a range of different factors can induce trap states and thus the distribution of traps is not expected to be the same for these different sources, as evident in the different types of distributions observed and used in literature. ${ }^{20,22,42,44}$ Here, we take a general approach and simply change the energetic disorder by swapping site energies with a fixed trap energy level, see Fig. 3. Activation energies associated with traps are found to be in the range of 0.05 to $0.4 \mathrm{eV}$ with the total trap densities ranging from $10^{21}$ to $10^{24} \mathrm{~m}^{-3},{ }^{20,22}$ which equates to one trap every $10^{3}-10^{6} \mathrm{~nm}^{3}$. Swapping site energies for fixed trap energy reduce the energetic disorder. However, in the supplementary material ${ }^{28}$ we show that for the aforementioned trap densities the trap population is essentially a small perturbation to the total energy landscape and the reduction in the standard deviation of energetic disorder is negligible. Electron transport in organic materials is frequently hindered by electron traps, while hole transport often shows trap-free behaviour. ${ }^{45}$ P3HT seems to be an exception as evidence for trapped holes has been reported, ${ }^{42,46}$ but the hole trap density (a)

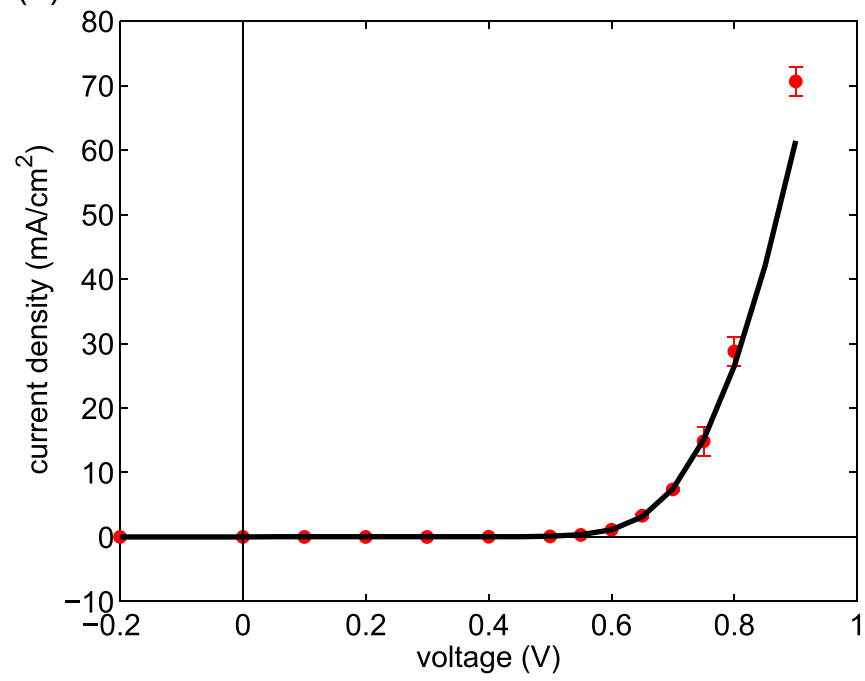

(b)

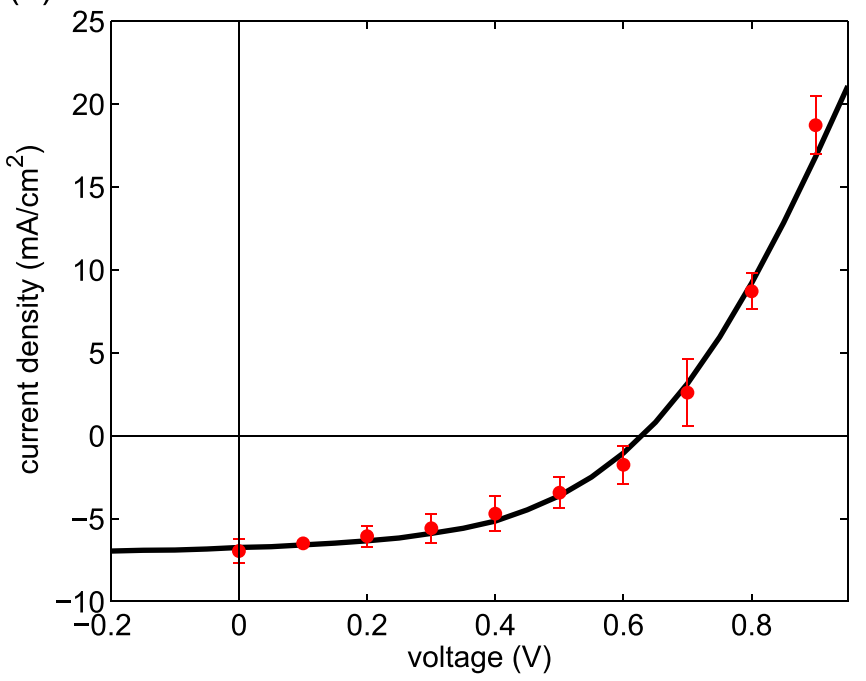

FIG. 2. (a) A measured dark I-V curve (black) and simulated dark I-V curve (red dots) with corresponding standard deviation. The thickness of the real and simulated BHJ device was approximately $50 \mathrm{~nm}$. (b) A measured I-V curve (black) and simulated I-V curve (red) under $1000 \mathrm{~W} / \mathrm{m}^{2}$ illumination. The thickness of the real and simulated BHJ device was approximately $100 \mathrm{~nm}$. 


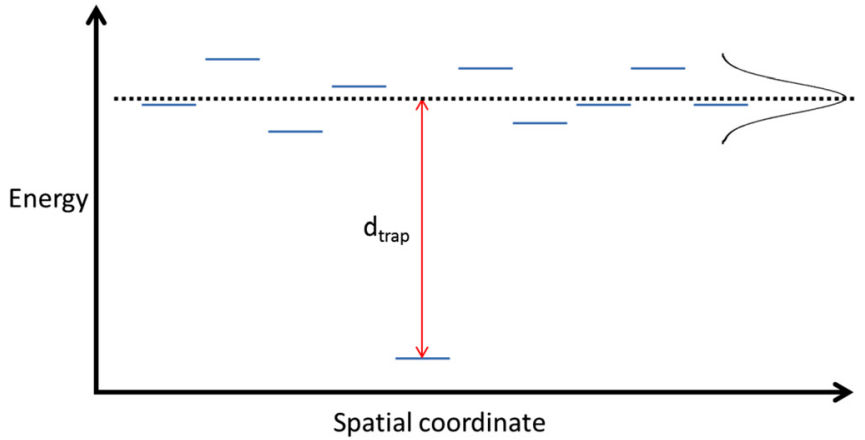

FIG. 3. Depiction of the energy landscape which encompasses Gaussian disorder and a trap state. Some of the initial energy levels which follow a Gaussian distribution are replaced by a trap site with a fixed energy below the average energy of the Gaussian, $d_{\text {trap }}$.

is much lower than the electron trap density. ${ }^{20}$ Considering the apparent dominance of electron traps over hole traps, we only simulate electron traps consistent with common materials like poly[2-methoxy-5-(2-ethylhexyloxy)-p-phenylenevinylene] (MEH-PPV), poly[9,9-didecaneuorene-alt-(bisthienylene) benzothiadiazole] (PF10TBT), poly[2,6-(4,4bis-(2-ethylhexyl)-4H-cyclopenta[2,1-b;3,4-b]-dithiophene)alt-4,7-(2,1,3-benzothiadiazole)] (PCPDTBT). ${ }^{45}$ These electron traps are introduced in the model by creating traps in the energy landscape of the electron donor phase with trap depth, $d_{\text {trap }}$, of up to $0.5 \mathrm{eV}$ in random locations with an average trap density, $n_{\text {trap }}$.

Exciton transport is also affected by energy traps. However, since excitons are a neutrally charged species, their transport is assumed not to be affected by the electric fields found in a working OPV device. Hence, excitons cannot produce the s-shaped anomaly that we investigated in this study. Nevertheless, the effect of energy traps on exciton dissociation has been investigated and is discussed in the supplementary material. ${ }^{28}$

\section{RESULTS AND DISCUSSION}

\section{A. Comparison of traps in the bulk of the active layer with traps at the electrode interfaces}

Given that electrode interface effects have already been identified as a source of s-shape behaviour, ${ }^{47}$ we introduced electron traps at sites that were immediately neighbouring either electrode. These interface traps are distinguished from trap sites that are not in contact with an electrode (bulk traps). In order to establish whether s-shaped I-V curves can be generated using our DMC approach, devices were simulated with a range of energy trap depths and densities. Fig. 4(a) shows the evolution of the I-V shape with increasing trap density while keeping the trap depth fixed at $0.2 \mathrm{eV}$. The black curve corresponds to a device without any traps and it performs best in terms of $J_{S C}, V_{O C}, F F$ and consequently also $P C E$. While $F F$ is affected the most by electron traps, a decrease in $J_{S C}$ and $V_{O C}$ is also seen, which agrees with experimental observations shown in Fig. 1. Arrows indicate how the I-V curves change with increasing trap density and show that the severity of the s-shape anomaly is more pronounced as the trap density increases. Likewise, the arrows in Fig. 4(b) indicate that an increase in trap depth, while keeping the density constant, also increases the severity of the anomaly. However, there is only a minor difference between the I-V curves corresponding to a trap depth of $0.4 \mathrm{eV}$ and $1 \mathrm{eV}$, which indicates that electrons are already efficiently trapped at the trapping sites for a trap depth of $0.4 \mathrm{eV}$. This saturation effect was also seen in the evolution of exciton diffusion with trap depth (see supplementary material ${ }^{28}$ ).

Having established, with DMC modelling, that interface traps give rise to s-shaped $\mathrm{I}-\mathrm{V}$ behaviour, we investigate the influence that bulk electron traps have on the J-V shape. Bulk traps were randomly created in the energy landscape but, in order to avoid interface traps, electron traps were not added to sites that were within $10 \mathrm{~nm}$ of the electrode. Various trap depths and densities were investigated and the results are shown in Fig. 5. I-V curves were obtained up to a trap density of $2 \times 10^{26} \mathrm{~m}^{-3}$, which is at least two orders of magnitude larger than the typical trap densities reported in the literature for P3HT:PCBM devices, ${ }^{20,22}$ but no s-shape was seen. In fact, the I-V curve corresponding to a trap density around the upper limit reported in the literature $\left(\sim 10^{24} \mathrm{~m}^{-3}\right)$ and trap depth of $0.2 \mathrm{eV}$ could not be distinguished from the I-V curve of a virtual device without traps (see blue and black curves in Fig. 5(c)). Even for a trap density of $2 \times 10^{26} \mathrm{~m}^{-3}$ and $d_{\text {trap }}=0.2 \mathrm{eV}$ (Fig. 5(a)), only a slight change around $V_{O C}$ was observed. On the other hand, (a)

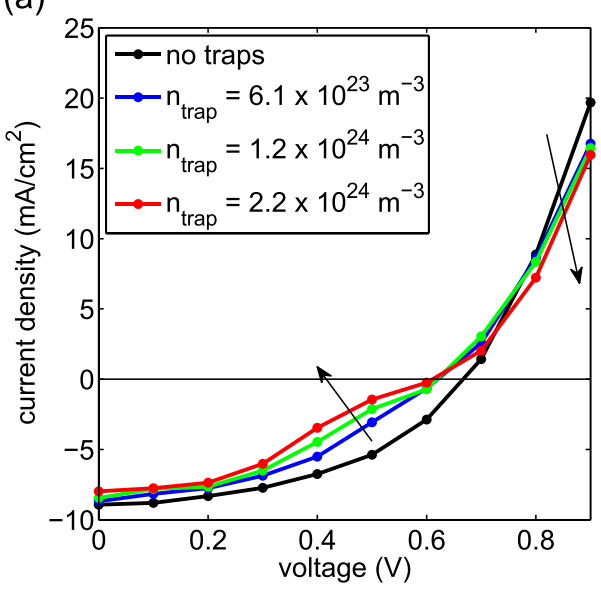

(b)

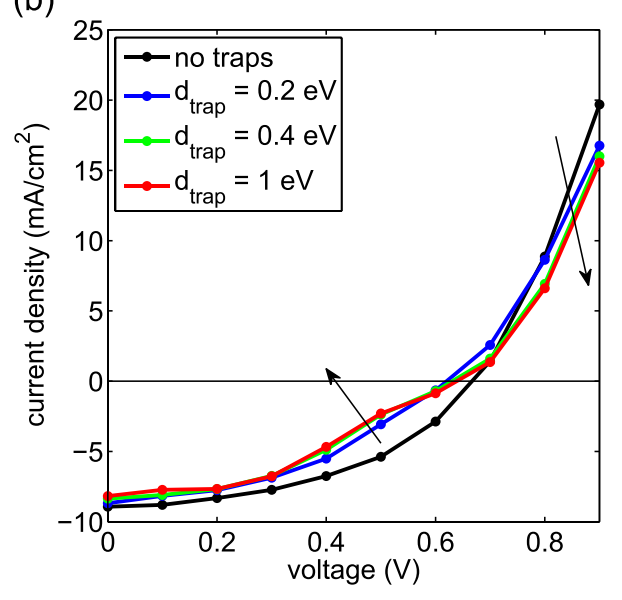

FIG. 4. (a) Current density as a function of voltage for various interface trap densities while the trap depth was fixed at $0.2 \mathrm{eV}$. (b) Current density as a function of voltage for various trap depths, while keeping the trap density fixed at $6.1 \times 10^{23} \mathrm{~m}^{-3}$. Arrows indicate the trend of the $\mathrm{I}-\mathrm{V}$ curves when increasing trap density (a) or trap depth (b). All virtual BHJ devices had an electron donor: electrode coverage of $50 \%$ and active layer thickness of $100 \mathrm{~nm}$. 
(a)

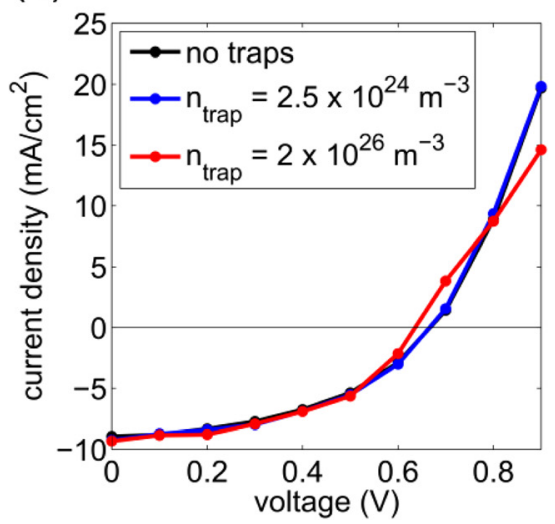

(b)

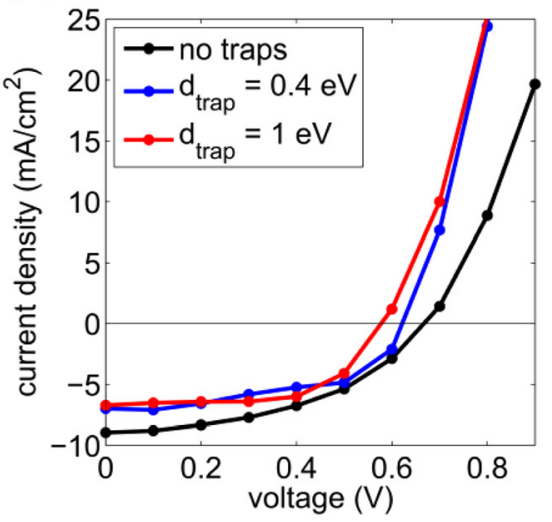

(c)

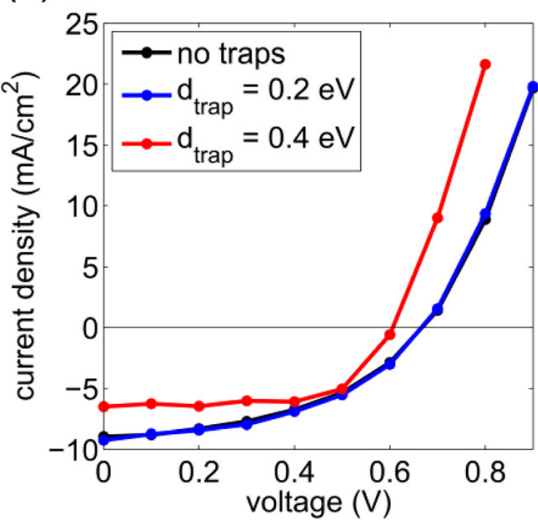

FIG. 5. (a) Simulated J-V curves for various bulk trap densities. The trap depth was fixed at 0.2 eV. (b) Simulated J-V curves for trap depths of 0.4 eV and $1 \mathrm{eV}$ while keeping the trap density fixed at $6.25 \times 10^{23} \mathrm{~m}^{-3}$. (c) $\mathrm{J}-\mathrm{V}$ curves for trap depths of $0.2 \mathrm{eV}$ and $0.4 \mathrm{eV}$ while keeping the trap density fixed at 2.5 $\times 10^{24} \mathrm{~m}^{-3}$. All virtual BHJ devices had an electron donor: electrode coverage of $50 \%$ and active layer thickness of $100 \mathrm{~nm}$.

for devices with interface traps (and $d_{\text {trap }}=0.2 \mathrm{eV}$ ) severely s-shaped I-V curves could be obtained with trap densities in the regime reported in the literature (Fig. 4(a)). Hence, DMC modelling suggests that, for realistic trap densities, no s-shape $\mathrm{I}-\mathrm{V}$ behaviour is induced by bulk traps. Deep traps $(0.4 \mathrm{eV}$ and $1 \mathrm{eV}$ ) reduce $\mathrm{J}_{\mathrm{sc}}$ and $\mathrm{V}_{\mathrm{oc}}$, but $\mathrm{FF}$ is hardly affected. Hence, while bulk traps do not induce s-shapes, they certainly reduce $P C E$ and should be avoided.

\section{B. Isolating the effect of compositional distribution at electrodes on I-V curves}

In the Introduction, we discussed that annealing removes s-shape effects by either changing the compositional distribution at the electrodes or by agglomeration of aluminium clusters. Experimentally, it is difficult to analyse these two effects separately; however, both effects are easily changed in the virtual OPV devices modelled by DMC. In Sec. III A, we showed that electron traps at the electrode interface, as would be caused by aluminium clusters, ${ }^{12}$ indeed cause s-shape I-V characteristics. Here, we discuss the effect of only changing the contact area between PCBM and the cathode and P3HT and the anode. In order to remove the effect of charge trapping clusters, virtual morphologies were used that did not contain any isolated islands, i.e., the morphologies consisted of a continuous inter-penetrating network of donor and acceptor material. In Fig. 6, simulated J-V curves are presented for three virtual devices that had morphologies of the same feature size, but different compositional phase distributions at the electrodes. For device 1, 50.3\% of the cathode was in contact with PCBM $(49.7 \%$ of the cathode was covered by P3HT) and $51.5 \%$ of the anode was in contact with P3HT (thus $48.5 \%$ of the anode was covered by PCBM). Device 2 had perfect capping layers at both electrodes, i.e., $100 \%$ of the anode is in contact with P3HT and $100 \%$ of the cathode is in contact with PCBM. Thus, device 1 has the same compositional distribution as an unannealed device and device 2 represents a device that is perfectly (a)

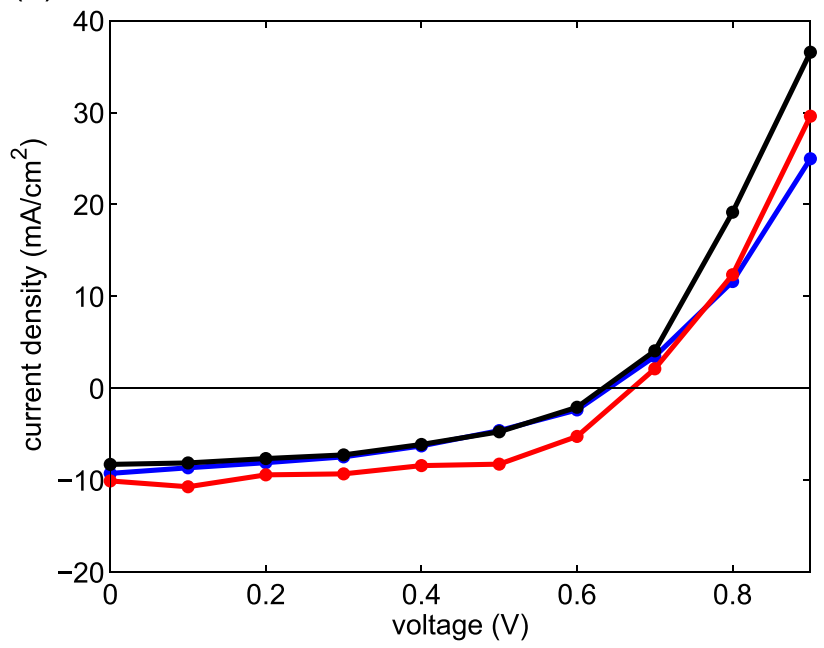

(b)

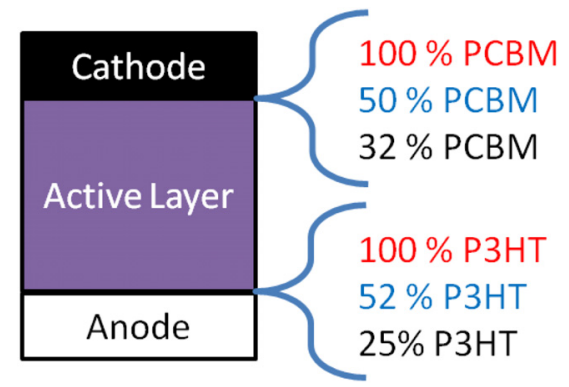

FIG. 6. (a) Simulated I-V curves for a device with (red) an active layer thickness of 100 nm and perfect blocking layers (blue) an active layer thickness of $100 \mathrm{~nm}$, where $50 \%$ of the cathode is in contact with PCBM and 52\% of the anode is in contact with P3HT, and (black) an active layer thickness of 85 nm, where $32 \%$ of the cathode is in contact with PCBM and $25 \%$ of the anode is in contact with P3HT. (b) Diagram depicting the P3HT and PCBM compositions at the electrodes. 
charge selective. ${ }^{11}$ Virtual devices 1 and 2 both had an active layer thickness of $100 \mathrm{~nm}$, while device 3 had an active layer thickness of $85 \mathrm{~nm}$. Device 3 had a PCBM-cathode contact percentage of $31.7 \%$ and a P3HT-anode contact percentage of $25.4 \%$. The donor-acceptor distribution is such that only a relatively small fraction of the cathode/anode is available for electron/hole extraction. Consequently, device 3 might be expected to exhibit s-shape behaviour. However, modelling results show no sign of s-shaped I-V characteristics (see black curve in Fig. 6(a)). Hence, the DMC modelling presented here indicates that s-shape behaviour cannot be induced solely by poor or inappropriate electrode interface composition. Indeed, these results suggest that the only way morphology can cause s-shape behaviour is through isolated islands of semiconductor material acting as charge traps near the electrodes, which agrees with the nanocluster explanation for s-shape behaviour of de Castro et al. ${ }^{12}$

For the simulation parameters used, $J_{S C}$ peaks at an active layer thickness of $85 \mathrm{~nm} .{ }^{11}$ Nevertheless, device 3 exhibits the lowest $J_{S C}$ due to a $\mathrm{P} 3 \mathrm{HT} / \mathrm{PCBM}$ electrode coverage that is not beneficial for charge extraction. Hence, while the chemical composition at the electrode does not induce s-shaped I-V curves, it does affect $J_{S C}, V_{O C}, F F$, and $P C E$. Since the thicknesses of devices 1 and 2 are the same, they can be directly compared. The morphology of the bulk is exactly the same for devices 1 and 2 and the only difference between the two devices is the presence of capping layers. As a result of the capping layers, a moderate increase is seen in $J_{S C}$ and $V_{O C}$, while a large increase is seen in FF and PCE compared to the corresponding values of the device without capping layers. Thus, the DMC model predicts that the presence of thin continuous capping layers at both electrodes considerably improves PV performance, which corresponds to the substantial performance increase seen experimentally when compared to structures without capping layers. ${ }^{13,48-50}$

\section{Injected charge carriers vs photo-generated charge carriers}

The total I-V characteristics describe the current flow in a device. In a photovoltaic device, these moving charge carriers are either photo-generated or injected at electrodes. I-V curves measured in the dark do not exhibit s-shaped I-V behaviour and thus the cause of the s-shape is related to photo-generated charges. The ratio of photo-generated charge carriers and injected charges can be directly manipulated by controlling the light intensity. Consequently, the severity of the inflection near $V_{O C}$ is expected to change with light intensity. Fig. 7 shows normalised experimental I-V curves for the same OPV device under various light intensities.

The severity of the anomalous behaviour is clearly seen to increase with light intensity. In order to quantify the magnitude of the inflection, i.e., the severity of the s-shape, we determined the slope at $V_{O C}$ for current normalised I-V curves, $S_{V o c}$. A small $S_{V o c}$ value indicates a very pronounced s-shape. Fig. 7(b) shows that $S_{V o c}$ decreases with light intensity and levels off at approximately $710 \mathrm{~W} / \mathrm{m}^{2}$, which further confirms that the s-shape is caused by photo-generated charges. Thus, from a modelling point of view, it is important to correctly estimate the charge injection and exciton generation rates.

Similar to the real experiment, a virtual experiment was conducted where the light intensity, i.e., exciton generation rate, was varied. A virtual device was chosen with interface traps that had a density of $1.2 \times 10^{24} \mathrm{~m}^{-3}$ and a depth of 0.2 $\mathrm{eV}$. The resulting I-V curves are shown in Fig. 8. Analogous to the experimental data, $V_{O C}$ decreased with light intensity, but $F F$ improved since the s-shape became progressively less pronounced. $S_{V o c}$ was also determined for the simulated data.

Even though the simulated curve contains fewer data points compared to the experimentally measured curves in Fig. 7 and model parameters were not fitted to match the experimental data, the same $S_{V o c}$ trend is observed with light intensity (Fig. 8(b)), which suggests that this light intensity behaviour is universal for all s-shaped I-V curves and provides further confidence in the predicted trends using DMC modelling.

\section{Charge recombination and s-shape severity}

Light intensity determines the charge carrier density, which in turn affects bimolecular recombination. ${ }^{38,51,52}$ In fact, the device corresponding to Fig. 7 exhibited significant bimolecular recombination as its $V_{O C}$ increased linearly with (a)

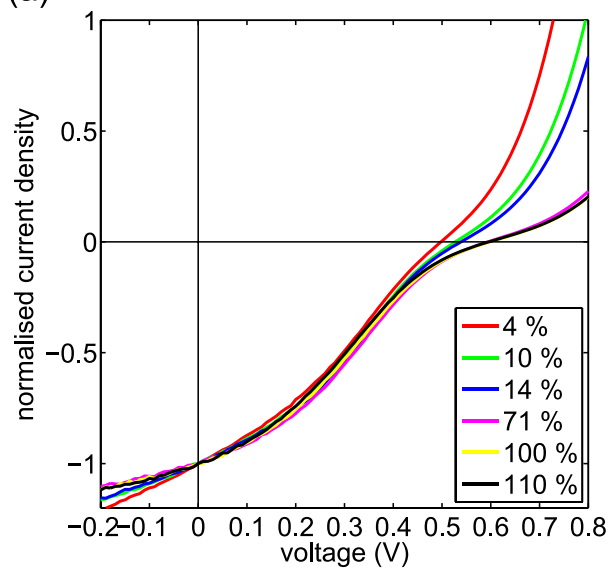

(b)

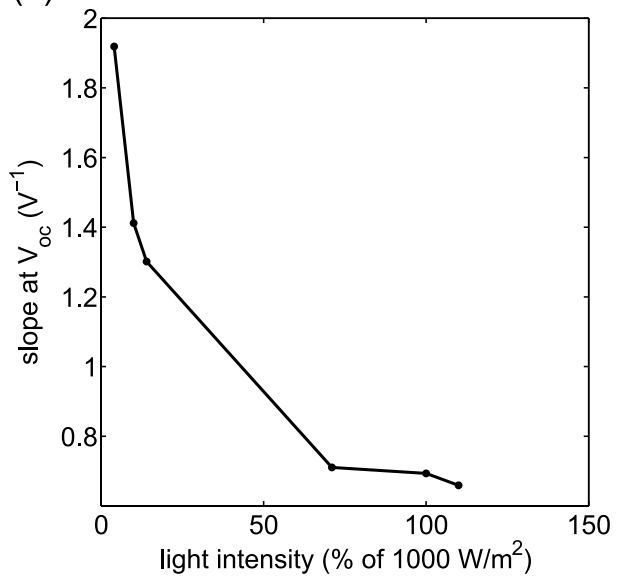

FIG. 7. (a) Normalised I-V curve of an s-shape exhibiting device measured (not simulated) under various light intensities as indicated in the legend (percentage of $1000 \mathrm{~W} / \mathrm{m}^{2}$ AM1.5 illumination). (b) $S_{V o c}$ of the normalised curves shown in (a) as a function of light intensity. 

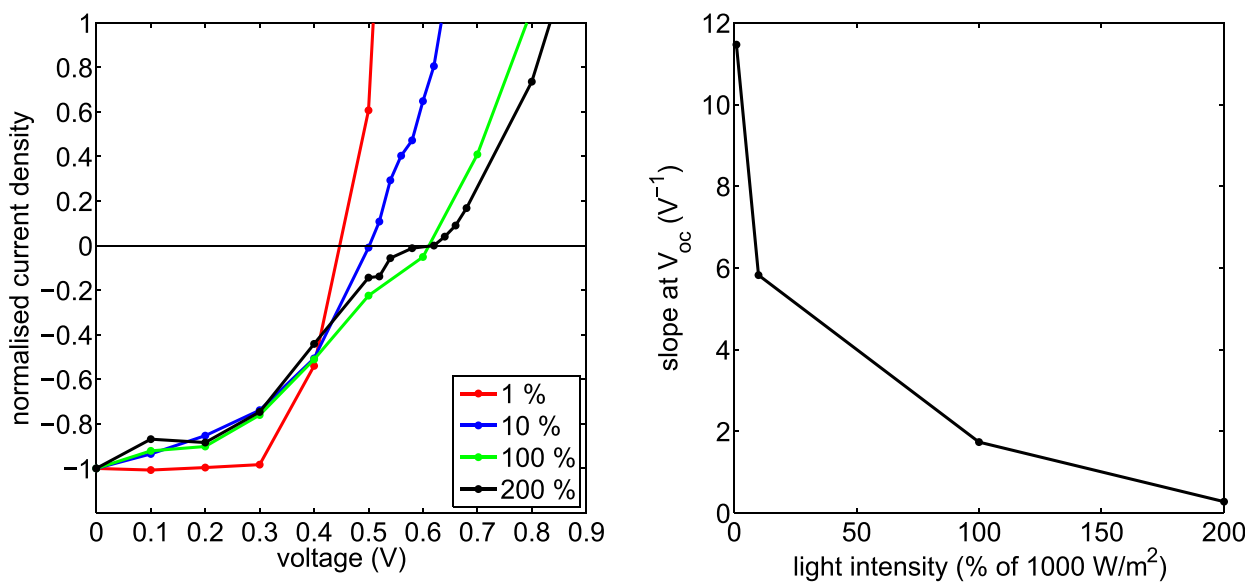

FIG. 8. (a) Simulated current normalised I-V curves for various light intensities (indicated in the legend as a percentage of $1000 \mathrm{~W} / \mathrm{m}^{2}$ ). (b) $S_{V o c}$ of normalised curves shown in (a) as a function of light intensity.

the logarithm of light intensity only up until $710 \mathrm{~W} / \mathrm{m}^{2}$, indicating that significant bimolecular recombination takes place for intensities above $710 \mathrm{~W} / \mathrm{m}^{2}$.

In Sec. III C, we established that s-shape behaviour is readily influenced by light intensity. Consequently, depending on the morphology and material system, bimolecular recombination may be expected to influence s-shape severity as well. In this section, we specifically investigate how the charge recombination rate influences the s-shape severity.

Fig. 9 shows J-V curves for a series of virtual devices where $k_{\text {rec }}$ was varied from $1 \mathrm{~s}^{-1}$ to $10^{10} \mathrm{~s}^{-1}$. As expected, $J_{S C}$ decreases as $k_{\text {rec }}$ increases due to more efficient charge recombination. When $k_{r e c}=1 \times 10^{10} \mathrm{~s}^{-1}$ almost all photogenerated charges recombine and the curve resembles a dark $\mathrm{I}-\mathrm{V}$ curve. From the series, it can be seen that the centre of the inflection (minimum of $d I / d V$ ) is not constrained by $V_{O C}$, i.e., for the low charge recombination devices, the centre of the inflection is not located at $V_{O C}$. To illustrate the location of the inflection with respect to $V_{O C}$, the derivative of the experimentally measured I-V curves in Fig. 10(a) is shown in Fig. 10(b).

Only s-shaped I-V curves have a minimum, which corresponds to the centre of the inflection. The location of the

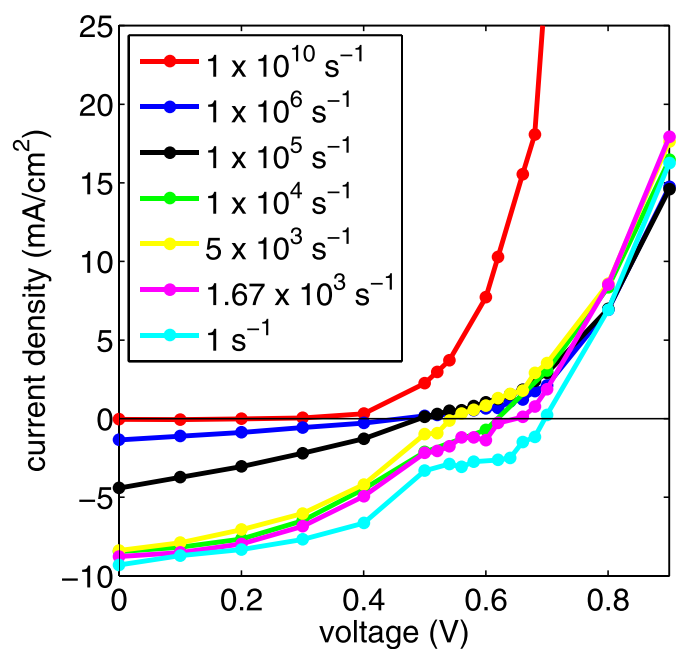

FIG. 9. J-V curves of a series of virtual devices where $k_{r e c}$ was changed. The legend indicates the corresponding $k_{r e c}$ values for each $\mathrm{J}-\mathrm{V}$ curve. inflection of the red curve in Fig. 10(a) does not coincide with its $V_{O C}$ (Fig. 10(b)), which is in contrast to the blue curve. As such, models where the centre of the inflection is fixed are not adequate to model all s-shaped curves.

$V_{O C}$ has been shown to decrease with increased charge recombination strength. ${ }^{51,53}$ The DMC simulations confirm this relationship as the $V_{O C}$ can clearly be seen to increase with decreasing $k_{r e c}$ in Fig. 9. The built-in field of the simulated devices would permit a maximum $V_{O C}$ of $0.72 \mathrm{~V}$ and is almost achieved for the device with the lowest $k_{\text {rec }}$. Unlike $V o c$, the s-shape severity seems to decrease with increasing $k_{\text {rec }}$. In other words, the s-shape is much more pronounced in devices with little recombination. The average time for which charge carriers are trapped does not change significantly when changing $k_{\text {rec }}$. Thus, the s-shape severity must be influenced by the number of free charge carriers available, which is affected both by light intensity (Figs. 7 and 8) and recombination rate (Fig. 9). DMC modelling predicts, somewhat counter-intuitively, that a system with very little bimolecular recombination is more prone to exhibit s-shape behaviour.

In order to understand better how charge recombination influences anomalous I-V curves, we analysed the two types of charge recombination that occur in a device: interface recombination $\left(\eta_{\text {int }}\right)$ and bulk recombination $\left(\eta_{\text {bulk }}\right){ }^{11}$ Interface recombination refers to charge carriers that are extracted at the opposite polarity electrode, while bulk recombination refers to recombination that occurs in the active layer (exciton, geminate, and bimolecular recombination).

Fig. 11 shows $\eta_{b u l k}, \eta_{\text {int }}$ and the total charge recombination efficiency $\left(\eta_{\text {rec }}=\eta_{\text {bulk }}+\eta_{\text {int }}\right)$ for a device with an s-shaped I-V curve and a non-anomalous I-V curve. In these plots, $\eta_{\text {bulk }}$ and $\eta_{\text {int }}$ can exceed unity, because injected charge carriers that are extracted are not distinguished from photogenerated charge carriers. $\eta_{\text {rec }}$ and $\eta_{\text {int }}$ can be seen to have the same shape as the I-V curve. $\eta_{\text {bulk }}$, on the other hand, does not resemble the shape of the I-V curve. Furthermore, there is only a marginal difference between $\eta_{\text {bulk }}$ of the s-shaped and that of the normal device. Hence, a comparison of $\eta_{\text {bulk }}$ and $\eta_{\text {int }}$ as a function of voltage (Figs. 11(a) and 11(b)) reveals that the s-shape of the I-V curves finds its origin in interface recombination. Despite the fact that injected charges, that are created at the interface, do not induce 
(a)

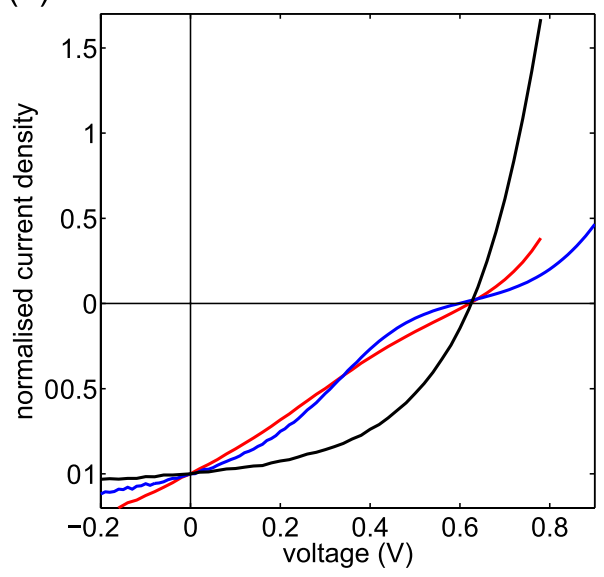

(b)

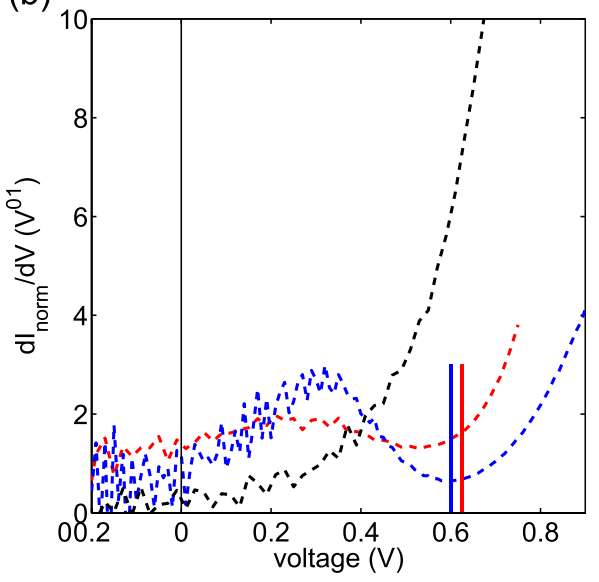

FIG. 10. (a) Current normalised experimentally measured I-V curve for three P3HT:PCBM devices. The corresponding derivative of the normalised $\mathrm{I}-\mathrm{V}$ curves is shown in (b) using the same colour (dashed curves) and also includes solid lines to indicate the $V_{O C}$ of the red and blue curves.
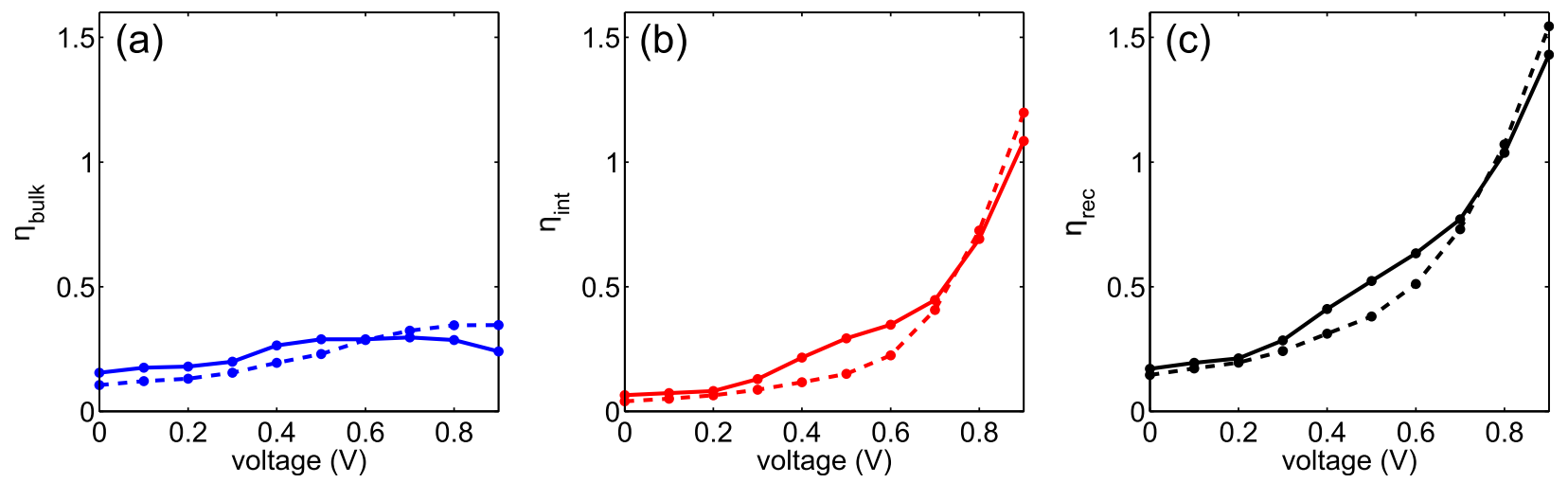

FIG. 11. (a) $\eta_{b u l k}$, (b) $\eta_{\text {int }}$, and (c) $\eta_{r e c}$ as a function of voltage for a virtual device with no traps and thus no s-shape I-V behaviour (dashed lines) and a virtual device with interface traps that exhibits an s-shaped I-V curve (solid lines).

anomalous I-V behaviour and photo-generated charge carriers, that are created in the bulk, are necessary to create s-shaped I-V curves, Fig. 11 demonstrates the importance of controlling electrode interface processes in order to prevent anomalous I-V behaviour.

\section{CONCLUSION}

We have used a DMC model to investigate the nature of s-shaped I-V curves in OPV devices. The modelling shows that electrode interface charge traps with realistic trap densities induce s-shaped curves, while bulk traps do not. As such, the electrode-active layer interface is crucial in preventing s-shaped curves. The s-shape anomaly becomes more pronounced with increasing interface trap depth and density. The ability to reproduce inefficient devices (in addition to efficient devices) highlights the all-around capability of DMC models to describe OPV devices.

Annealing procedures influence several film properties including vertical phase segregation and clustering of isolated aluminium domains. These latter two effects have been proposed to induce s-shaped I-V characteristics. It is difficult to distinguish between the two effects experimentally because they both change simultaneously during annealing treatments. However, DMC modelling allowed us to investigate both effects separately. As shown by modelling, electron traps that may be formed by isolated aluminium clusters can certainly induce s-shaped curves. On the other hand, morphologies with no charge traps, but with different vertical phase compositions were not seen to induce s-shaped behaviour. Hence, DMC modelling supports the idea that $\mathrm{S}$-shaped I-V behaviour is induced by aluminium nanoclusters as opposed to vertical phase segregation. The only way that morphology can induce s-shaped I-V behaviour is when isolated islands of either phase are present near the electrodes. $J_{S C}, V_{O C}, F F$, and $P C E$ were influenced by the phase distribution at the electrodes, showing that it is still important to control the phase composition even though it does not cause the s-shape anomaly. From a technical standpoint, care should be taken when evaporatively depositing interface layers or electrodes. The compatibility of materials and the deposition rate should be tuned to avoid embedded nanoclusters.

Interestingly, charge carriers that are injected from the electrode into the active layer do not induce s-shaped behaviour. Rather, photo-generated charge carriers are necessary to observe the s-shape anomaly. Furthermore, both simulated and experimental data show that the anomaly becomes more pronounced as the free charge carrier density increases, which is both influenced by light intensity and charge recombination. While it is known that charge recombination increases with light intensity, the DMC simulations indicate that charge recombination alone does not cause s-shape I-V characteristics. In fact, the s-shape become less pronounced 
with increasing bulk charge recombination, which suggests that efficient OPV material systems are more susceptible to electrode interface effects.

Experimental and simulated data show that the centre of the inflection does not always coincide with $V_{O C}$. Consequently, models where the centre of the inflection is fixed are not adequate to describe all s-shaped I-V curves. In the DMC model, the centre of the inflection could be changed with respect to $V_{O C}$ by tuning bulk charge recombination. The DMC model allowed us to distinguish between bulk recombination and interface recombination (at the electrode). The latter is shown to induce s-shape I-V curves, again highlighting the importance of controlling the electrode interface.

It is difficult to completely avoid trap states in disordered organic materials. However, the DMC results show that only traps near the electrode interfaces have to be avoided to prevent s-shaped IV behaviour. As such, appropriate interface layers may assist in avoiding trap states from forming near the electrodes, thus preventing the s-shape anomaly.

\section{ACKNOWLEDGMENTS}

This work was supported by the Australian Government, through the Australian Renewable Energy Agency. This work was performed in part at the Materials node of the Australian National Fabrication Facility, which is a company established under the National Collaborative Research Infrastructure Strategy to provide nano and microfabrication facilities for Australia's researchers.

${ }^{1}$ L. J. A. Koster, E. C. P. Smits, V. D. Mihailetchi, and P. W. M. Blom, Phys. Rev. B 72, 085205 (2005).

${ }^{2}$ A. Cheknane, H. S. Hilal, F. Djeffal, B. Benyoucef, and J.-P. Charles, Microelectron. J. 39, 1173 (2008).

${ }^{3}$ J. Wagner, M. Gruber, A. Wilke, Y. Tanaka, K. Topczak, A. Steindamm, U. Hoürmann, A. Opitz, Y. Nakayama, H. Ishii, J. Pflaum, N. Koch, and W. Bruütting, J. Appl. Phys. 111, 054509 (2012).

${ }^{4}$ B. Ecker, H. Egelhaaf, R. Steim, J. Parisi, and E. von Hauff, J. Phys. Chem. C 116, 16333 (2012).

${ }^{5}$ A. Wagenpfahl, D. Rauh, M. Binder, C. Deibel, and V. Dyakonov, Phys. Rev. B 82, 115306 (2010).

${ }^{6}$ B. Tremolet de Villers, C. J. Tassone, S. H. Tolbert, and B. J. Schwartz, J. Phys. Chem. C 113, 18978 (2009).

${ }^{7}$ G. del Pozo, B. Romero, and B. Arredondo, Sol. Energy Mater. Sol. Cells 104, 81 (2012).

${ }^{8}$ B. Xue, B. Vaughan, C. Poh, K. B. Burke, L. Thomsen, A. Stapleton, X. Zhou, G. W. Bryant, W. Belcher, and P. C. Dastoor, J. Phys. Chem. C 114, 15797 (2010).

${ }^{9}$ A. J. Parnell, A. D. F. Dunbar, A. J. Pearson, P. A. Staniec, A. J. C. Dennison, H. Hamamatsu, M. W. A. Skoda, D. G. Lidzey, and R. A. L. Jones, Adv. Mater. 22, 2444 (2010)

${ }^{10}$ Z. Xu, L. M. Chen, G. Yang, C. H. Huang, J. Hou, Y. Wu, G. Li, C. S. Hsu, and Y. Yang, Adv. Funct. Mater. 19, 1227 (2009).

${ }^{11}$ K. Feron, C. J. Fell, L. J. Rozanski, B. B. Gong, N. Nicolaidis, W. J. Belcher, X. Zhou, E. Sesa, B. V. King, and P. C. Dastoor, Appl. Phys. Lett. 101, 193306 (2012).

${ }^{12}$ F. A. de Castro, J. Heier, F. Nüesch, and R. Hany, IEEE J. Sel. Top. Quantum Electron. 16, 1690 (2010).

${ }^{13}$ R. Steim, F. R. Kogler, and C. J. Brabec, J. Mater. Chem. 20, 2499 (2010).

${ }^{14}$ G. Parthasarathy, C. Adachi, P. E. Burrows, and S. R. Forrest, Appl. Phys. Lett. 76, 2128 (2000).

${ }^{15}$ W. Tress, A. Petrich, M. Hummert, M. Hein, K. Leo, and M. Riede, Appl. Phys. Lett. 98, 063301 (2011).
${ }^{16}$ B. Romero, G. del Pozo, and B. Arredondo, Sol. Energy 86, 3026 (2012).

${ }^{17}$ A. Kumar, S. Sista, and Y. Yang, J. Appl. Phys. 105, 094512 (2009).

${ }^{18}$ N. Koch, G. Leising, A. Rajagopal, and J.-J. Pireaux, Conjugated Polymer and Molecular Interfaces, 1st ed. (Marcel Dekker, New York, 2002), pp. 218-219.

${ }^{19}$ H. Gommans, B. Verreet, B. P. Rand, R. Muller, J. Poortmans, P. Heremans, and J. Genoe, Adv. Funct. Mater. 18, 3686 (2008).

${ }^{20}$ J. Schafferhans, A. Baumann, A. Wagenpfahl, C. Deibel, and V. Dyakonov, Org. Electron. 11, 1693 (2010).

${ }^{21}$ M. M. Mandoc, F. B. Kooistra, J. C. Hummelen, B. de Boer, and P. W. M. Blom, Appl. Phys. Lett. 91, 263505 (2007).

${ }^{22}$ N. Giebink, G. Wiederrecht, M. Wasielewski, and S. Forrest, Phys. Rev. B 82, 155305 (2010).

${ }^{23}$ K. Feron, W. J. Belcher, C. J. Fell, and P. C. Dastoor, Int. J. Mol. Sci. 13, 17019 (2012)

${ }^{24}$ P. K. Watkins, A. B. Walker, and G. L. B. Verschoor, Nano Lett. 5, 1814 (2005).

${ }^{25}$ R. G. E. Kimber, E. N. Wright, S. E. J. O'Kane, A. B. Walker, and J. C. Blakesley, Phys. Rev. B 86, 235206 (2012).

${ }^{26}$ K. Feron, X. Zhou, W. J. Belcher, and P. C. Dastoor, J. Appl. Phys. 111, 044510 (2012).

${ }^{27}$ P. Peumans, S. Uchida, and S. R. Forrest, Nature 425, 158 (2003).

${ }^{28}$ See supplementary material at http://dx.doi.org/10.1063/1.4903530 for the morphology generation methodology, evaluation of the cut-off approximation for Coulomb interaction, effect of energy traps on exciton transport, and evaluation of the fixed trap depth approximation.

${ }^{29}$ N. C. Nicolaidis, B. S. Routley, J. L. Holdsworth, W. J. Belcher, X. Zhou, and P. C. Dastoor, J. Phys. Chem. C 115, 7801 (2011).

${ }^{30}$ T. Förster, Ann. Phys. 437, 55 (1948).

${ }^{31}$ R. A. Marcus, J. Chem. Phys. 24, 966 (1956).

${ }^{32}$ M. Casalegno, G. Raos, and R. Po, J. Chem. Phys. 132, 094705 (2010).

${ }^{33}$ P. Yu, D. Mencaraglia, A. Darga, A. Migan, R. Rabdbeh, B. Ratier, and A. Moliton, Phys. Status Solidi C 7, 1000 (2010).

${ }^{34}$ P. Peumans, A. Yakimov, and S. R. Forrest, J. Appl. Phys. 93, 3693 (2003).

${ }^{35}$ P. E. Shaw, A. Ruseckas, and I. D. W. Samuel, Adv. Mater. 20, 3516 (2008).

${ }^{36}$ S. Cook, H. Ohkita, J. R. Durrant, Y. Kim, J. J. Benson-Smith, J. Nelson, and D. D. C. Bradley, Appl. Phys. Lett. 89, 101128 (2006).

${ }^{37}$ L. Meng, Y. Shang, Q. Li, Y. Li, X. Zhan, Z. Shuai, R. G. E. Kimber, and A. B. Walker, J. Phys. Chem. B 114, 36 (2010).

${ }^{38}$ R. A. Marsh, C. Groves, and N. C. Greenham, J. Appl. Phys. 101, 083509 (2007).

${ }^{39}$ D. C. Coffey, B. W. Larson, A. W. Hains, J. B. Whitaker, N. Kopidakis, O. V. Boltalina, S. H. Strauss, and G. Rumbles, J. Phys. Chem. C 116, 8916 (2012).

${ }^{40}$ Y. S. Eo, H. W. Rhee, B. D. Chin, and J.-W. Yu, Synth. Met. 159, 1910 (2009).

${ }^{41}$ A. Baumann, J. Lorrmann, C. Deibel, and V. Dyakonov, Appl. Phys. Lett. 93, 252104 (2008).

${ }^{42}$ R. C. I. MacKenzie, T. Kirchartz, G. F. A. Dibb, and J. Nelson, J. Phys. Chem. C 115, 9806 (2011).

${ }^{43}$ A. Sharma, S. Yadav, P. Kumar, S. Ray Chaudhuri, and S. Ghosh, Appl. Phys. Lett. 102, 143301 (2013).

${ }^{44}$ H. T. Nicolai, M. M. Mandoc, and P. W. M. Blom, Phys. Rev. B 83, 195204 (2011).

${ }^{45}$ G.-J. A. H. Wetzelaer, M. Kuik, and P. W. M. Blom, Adv. Energy Mater. 2, 1232 (2012).

${ }^{46}$ Z. Chiguvare and V. Dyakonov, Phys. Rev. B 70, 235207 (2004).

${ }^{47}$ S. Trost, K. Zilberberg, A. Behrendt, A. Polywka, P. Görrn, P. Reckers, J. Maibach, T. Mayer, and T. Riedl, Adv. Energy Mater. 3, 1437 (2013).

${ }^{48}$ M. Riede, T. Mueller, W. Tress, R. Schueppel, and K. Leo, Nanotechnology 19, 424001 (2008).

${ }^{49}$ J. Xue, B. P. Rand, S. Uchida, and S. R. Forrest, J. Appl. Phys. 98, 124903 (2005).

${ }^{50}$ J. Drechsel, B. Männig, F. Kozlowski, D. Gebeyehu, A. Werner, M. Koch, K. Leo, and M. Pfeiffer, Thin Solid Films 451-452, 515 (2004).

${ }^{51}$ L. J. A. Koster, V. D. Mihailetchi, R. Ramaker, and P. W. M. Blom, Appl. Phys. Lett. 86, 123509 (2005).

${ }^{52}$ L. J. A. Koster, V. D. Mihailetchi, H. Xie, and P. W. M. Blom, Appl. Phys. Lett. 87, 203502 (2005).

${ }^{53}$ B. Ray, M. S. Lundstrom, and M. A. Alam, Appl. Phys. Lett. 100, 013307 (2012). 